\title{
Annihilation of Charged Particles
}

\author{
I. Ispolatov and P. L. Krapivsky* \\ Center for Polymer Studies and Department of Physics \\ Boston University, Boston, MA 02215
}

\begin{abstract}
The kinetics of irreversible annihilation of charged particles performing overdamped motion induced by long-range interaction force, $F(r) \sim$ $r^{-\lambda}$, is investigated. The system exhibits rich kinetic behaviors depending on the force exponent $\lambda$. In one dimension we find that the densities decay as $t^{-1 /(2+\lambda)}$ and $t^{-1 /(1+2 \lambda)}$ when $\lambda>1$ and $1 / 2<\lambda<1$, respectively, with logarithmic correction at $\lambda=1$. For $\lambda \leq 1 / 2$, the asymptotic behavior is shown to be dependent on system size.
\end{abstract}

PACS number(s): 64.60.Cn, 64.60.My

^ Present address: Courant Institute of Mathematical Sciences, New York University, New York, NY 10012 


\section{INTRODUCTION}

The kinetics of two-species diffusion-controlled annihilation reaction, $A+B \rightarrow 0$, between uncharged particles has been a subject of extensive research for almost 20 years [1]. For sufficiently low spatial dimension, $d<4$, even under homogeneous initial conditions, large-scale heterogeneities arise that invalidate classical kinetic laws. Much less is known about annihilation reaction between charged particles with long-range power-law interaction, $F(r) \sim r^{-\lambda}$. An important case of Coulomb interaction $(\lambda=d-1$ in $d$ dimensions $)$ has been treated in a few studies [2-5] for $d=2$ and 3 . However, some of these works were based on unjustified approximations while others were based solely on numerical simulations, so their results are also uncertain. (Note that even for annihilation of uncharged particles in three dimensions the asymptotic regime is hardly reached on modern computers). We, therefore, see that the Coulomb case still deserves further investigation. Other values of the interaction exponent $\lambda$ also naturally appear in applications with particles being dipoles, defects, vortices, monopoles, disclinations, etc. One important example is the quench of a one-dimensional Ising system from a disordered state to an ordered state. If spins interact via long-range potential [6], the Hamiltonian may be expressed in terms of interacting domain walls [7]. There are two types of domain walls in the system, the domain walls with "up" spins to the right and "down" to the left ( $A$-walls), and vice versa ( $B$-walls). Thus, an alternating domain wall sequence $\ldots A B A B A B \ldots$ is formed. Domain walls annihilate upon colliding, $A+B \rightarrow 0$, but since the alternating structure persists in time, the reaction process is, in fact, equivalent to the single-species annihilation, $D+D \rightarrow 0$. This system has been recently investigated [7-9], and it was shown that particle concentration decays as $t^{-1 /(1+\lambda)}$.

In this paper we consider a truly two-species annihilation model where the initial 
distribution of interacting particles ("charges") is random (Poissonian). The forces between

charges are assumed to be proportional to $r^{-\lambda}$, with similar charges repelling each other and dissimilar attracting each other. Compared to the single-species case, the two-species annihilation exhibits more rich kinetic behavior including the dependence on the system size. In this study we focus on one-dimensional systems which allow us to find rather convincing numerical support for our scaling predictions.

The paper has the following structure: In the next Section we formally introduce the model, an ensemble of interacting particles in one dimension with overdamped dynamics. Then, relying on heuristic arguments, we obtain density decay exponents for different values of $\lambda$. In Section III, we present the results of numerical simulations. Finally, in Section IV we discuss possible generalizations of the model including higher dimensionality and ballistic motion, and make general conclusions.

\section{THE MODEL AND SCALING ARGUMENTS}

We consider two-species systems containing $A$ - and $B$-type particles with charges +1 and -1 for "particles" and "antiparticles", respectively. Particles of both species move continuously in one dimension and interact via long-range force, $F=q q^{\prime} / r^{\lambda}$ (for charges $q$ and $q^{\prime}$ separated by the distance $r$ ). Initially, $A$ - and $B$-type particles are randomly distributed with equal concentrations, for simplicity we put them equal to 1 .

Total force acting on the $i^{\text {th }}$ particle is equal to a sum of pairwise forces:

$$
F_{i}=q_{i} \sum_{j \neq i} \frac{q_{j}\left(x_{i}-x_{j}\right)}{\left|x_{i}-x_{j}\right|^{\lambda+1}},
$$

where $q_{k}= \pm 1$ and $x_{k}$ are charge and coordinate of the $k^{\text {th }}$ particle. We will ignore particle inertia; i. e., motion of particles is assumed to be overdamped. Therefore, the 
velocity of each particle $v_{i}$ is proportional to the total force $F_{i}$ acting on it, $v_{i}=\mu F_{i}$. (In the following, we set the mobility $\mu$ equal to 1 ). We will also ignore particle diffusion; that is, we will assume that the drift dominates the random walk effects. When two dissimilar particles collide, both of them irreversibly disappear; collisions between particles of the same species are impossible because of repulsion. To summarize, we consider two-species annihilation of particles undergoing overdamped noiseless motion. We will see that for a sufficiently large force exponent $(\lambda>4$ in one dimension), the noise actually dominates the drift, and thus well-known diffusion-controlled kinetic behavior [1] emerges. However, for small $\lambda$, we expect that the long-time behavior is correctly described by our noiseless model. We will also briefly discuss a model where the motion is ballistic (Sec.V).

Let us now consider time evolution of the system. We cannot a priori expect that a mean-field description holds in low dimensions, especially in one dimension. Remember that the breakdown of the mean-field behavior in reaction-diffusion models is generally attributed to the formation of single-species domains [1]. We assume that the same takes place in our model (at least in one dimension, the formation of domains is inevitable).

Suppose that at time $t$ the length of a typical single-species domain is $L(t)$. It means that an average number of particles in such a domain is equal to initial imbalance of majority and minority species on the length $L(t)$, which for Poissonian initial distribution with the density one is of the order of $\sqrt{L(t)}$. Therefore, the concentration $n(t)$ in a typical domain behaves as

$$
n(t) \sim 1 / \sqrt{L(t)}
$$

To get an insight on how a typical domain length changes in time, we consider motion of a single particle $\tilde{A}$ on a domain edge (Fig. 1). Here we assume that the system is entirely formed of well-defined domains of typical length $L$. The total force acting on $\tilde{A}$ from the 
particles on its left may be evaluated as:

$$
F \sim \sum_{j=1}^{M} \frac{1}{x_{j}^{\lambda}}-\sum_{j=1}^{M} \frac{1}{\left(x_{j}+L\right)^{\lambda}}+\sum_{j=1}^{M} \frac{1}{\left(x_{j}+2 L\right)^{\lambda}}-\cdots .
$$

Here $M$ is a typical number of particles in a domain, $M \sim \sqrt{L}$. Each sum in the left-hand side of Eq. (3) expresses a contribution to the net force from a particular domain; to get the total force these contributions have been added. It is clear that the force exerted on $\tilde{A}$ from all the particles to the right may be calculated in exactly the same way. To simplify the matter even further we assume that $x_{j} \simeq R \times j$, where $R$ is an average interparticle separation; $R=L / M$. Rewriting Eq. (3) through $R$ and $M$ gives

$$
F \sim \frac{1}{R^{\lambda}}\left[\sum_{j=1}^{M} \frac{1}{j^{\lambda}}-\sum_{j=1}^{M} \frac{1}{(j+M)^{\lambda}}+\sum_{j=1}^{M} \frac{1}{(j+2 M)^{\lambda}}-\cdots\right]
$$

Depending on the value of the force exponent $\lambda$, different situations appear. For $\lambda>1$, the first sum converges to a finite value for $M \rightarrow \infty$ while the other sums approach to zero as $M^{-(\lambda-1)}$ which means that only charges from the left and right nearest neighbor domains essentially contribute to the total force. For $0<\lambda<1$, all sums diverge as $M^{1-\lambda}$ as $M \rightarrow \infty$. The total force, being the sum of monotonically decreasing signalternating terms, is of order of the contribution of the first domain. In the borderline case $\lambda=1$, the first sum diverges as $\log M$ while the others terms are monotonically decreasing, sign-alternating, and finite. The dominate contribution is again provided by the nearest domains. To sum up,

$$
F \sim \frac{1}{R^{\lambda}} \times\left\{\begin{array}{lll}
M^{1-\lambda} & \text { if } & 0<\lambda<1 \\
\log M & \text { if } & \lambda=1 \\
1 & \text { if } & \lambda>1
\end{array}\right.
$$

On the other hand, a typical rate of change of the domain length is of order of the 
velocity of any of its edges. Recalling that $R \sim M \sim \sqrt{L}$, we obtain

$$
d L / d t \sim F \sim\left\{\begin{array}{llc}
L^{1 / 2-\lambda} & \text { if } & 0<\lambda<1 \\
L^{-1 / 2} \log L & \text { if } & \lambda=1 \\
L^{-\lambda / 2} & \text { if } & \lambda>1 .
\end{array}\right.
$$

Solving (6) for $L(t)$ and using relation (2), we finally write for the density decay asymptotics:

$$
n(t) \sim\left\{\begin{array}{lll}
t^{-\frac{1}{1+2 \lambda}} & \text { if } & 0<\lambda<1, \\
(t \log t)^{-\frac{1}{3}} & \text { if } & \lambda=1 \\
t^{-\frac{1}{2+\lambda}} & \text { if } & \lambda>1 .
\end{array}\right.
$$

These results are, in fact, correct only for $1 / 2 \leq \lambda \leq 2$. The upper bound follows from comparison of the random walk length, $L_{\mathrm{RW}} \sim t^{1 / 2}$, with the drift length, $L \sim t^{2 /(2+\lambda)}$ when $\lambda>1$. For $\lambda>2, L_{\mathrm{RW}} \gg L$, so a pair of charges can escape annihilation through a random walk, and therefore the diffusion controls the dynamics. Thus, for $\lambda>2$, the diffusion-controlled asymptotic behavior, $n \sim t^{-1 / 4}$, is expected. The lower bound stems from the fact that an average force acting on any particle in the infinite-particles 1D system becomes infinite for $\lambda \leq 1 / 2$. It can be shown rigorously by deriving a Holtsmarklike [10] force distribution (we leave it for Appendix A). Here, we provide more qualitative arguments which take into account the finiteness of the system. First, we note that in calculation of the total force in (3), we implicitly assumed that the system is perfectly ordered - it consists of similar domains of $A$ and $B$ particles; $i$. e., the total charge of the first domain is equal, up to the sign, to the total charge of the second domain, etc. In particular, it means that for a system depicted in Fig. 1, the overall charge to the left of the test particle $\tilde{A}$ is -1 , and the overall charge to the right is zero. However, this picture is a "mean-field" in spirit; hence, it can lead to erroneous results for truly random systems. Fluctuations in initial charge distribution in the system with $N$ particles produce the net 
charge of the order $\sqrt{N}$ to the left and to the right of the test particle. Qualitatively, we can estimate the effect of charge imbalance by putting $\sqrt{N}$ equidistant charges of one sign to the right and the same amount of the opposite charges to the left. (When we consider finite systems of charges we implicitly assume that they satisfy the neutrality condition; generally, the total net charge determines the long-time behavior). The initial size of the system is $N$, so the distance between nearest charges left in the system is $\sqrt{N}$; thus, the force $F_{N}$ due to the charge imbalance is

$$
F_{N} \sim \frac{1}{N^{\lambda / 2}} \sum_{j=1}^{\sqrt{N}} \frac{1}{j^{\lambda}} \sim N^{-\lambda+1 / 2}
$$

While $F_{N} \rightarrow 0$ as $N \rightarrow \infty$, this force does not affect the dynamics of the model. Thus, for $\lambda>1 / 2$ our previous estimate, $F \sim L^{1 / 2-\lambda}$, gives the dominate contribution, and the size-independent dynamics (7) emerges. However, for $\lambda<1 / 2$ the total force acting on a particle grows with system size even for overall neutral systems; therefore, it should dominate over the "regular" force (3) and control the dynamics of the system. It seems reasonable to assume that at the early stages of time evolution, when the distribution of the particles is still almost random, the motion of domain edges is controlled by the force (8). Repeating the steps used in deriving Eq. (7), with $F \sim N^{1 / 2-\lambda}$ instead of Eq. (6), we obtain for the density decay

$$
n(t) \sim N^{\frac{2 \lambda-1}{4}} t^{-\frac{1}{2}}
$$

However, this estimate may become inapplicable on the later stages of evolution. Indeed, the dynamics described by Eq. (9) is extremely fast since it is size-dependent, so the charge distribution that emerges can be significantly different from the Poissonian; as a result, our assumption about the type of randomness of the particle distribution could become less and less appropriate. 


\section{SIMULATION RESULTS}

To check our heuristic predictions, we have performed numerical simulations for $\lambda=$ $1,0.75,0.5,0.25$, and 0 (the Coulomb case $\lambda=0$ turns out to be special; it will be discussed separately in Appendix A). Our system initially consisted of 10000 particles of each species randomly distributed with concentration 1 . First, the net force (1) is calculated for each particle. We compute all the forces directly without applying any multipole-like expansion that could be useful in many dimensions [12]. With the particle velocity equal to the total force, we employ a simple Euler update procedure for each time step: $\Delta x_{i}=F_{i} \Delta t$. The selection of time interval $\Delta t$ was merely experimental. Since on the last stages of evolution simulations run very fast (few particles are left), we, unlike [7-8], keep $\Delta t$ constant during a run. Finally the results are averaged over 10 runs. The selection of boundary conditions does not seem to affect the results of simulations of the two-component system with overall neutrality, except for, maybe, its latest stages. We ran the simulations for $\lambda=0.25$ with both periodic and open boundary conditions; the results for concentration coincided within a statistical error. Hence, we use open boundary conditions for all further simulations.

In Fig. 2 we plot, for various $\lambda$, the concentration of either species vs. time. As we anticipated, for $1 / 2<\lambda$ our predictions for the decay of concentration are in good agreement with the results of simulation. Indeed, the average slope of $\log (n(t)) v s . \log t$

plot for $\lambda=1$ is -0.322 [the heuristic argument gives $(t \log t)^{-1 / 3}$ ], and -0.401 (compared to $-2 / 5)$ for $\lambda=3 / 4$. Performing numerical simulations with a twice smaller system (5000 pairs of particles), we did not find any significant system size dependence for these values of $\lambda$. As for $\lambda \leq 1 / 2$, no power-law behavior can be observed for density decay. However, at the early stages of evolution, local exponents are close to $-1 / 2$ as it follows from Eq. (9); as time goes on, the density decay rate increases. 


\section{DISCUSSION}

Considering the dynamics of domain interfaces and using simple heuristic arguments, we have predicted the asymptotic density decay in a two-species annihilation reaction system with long-range interaction and overdamped motion. This approach can be generalized on similar systems in an arbitrary spatial dimension $d$. Following the line of reasoning described in Sec. II, one can obtain:

$$
n(t) \sim \frac{1}{\sqrt{L^{d}}} \sim\left\{\begin{array}{lll}
t^{-\frac{d}{2-d+2 \lambda}} & \text { if } & d / 2 \leq \lambda<d, \\
(t \log t)^{-\frac{d}{2+d}} & \text { if } & \lambda=d, \\
t^{-\frac{d}{2+\lambda}} & \text { if } & \lambda>d .
\end{array}\right.
$$

For $\lambda<d / 2$, we expect size-dependent kinetics. For $\lambda>2$, as in the $1 d$ case, let us compare diffusion and drift length scales, $L_{\mathrm{RW}} \sim t^{1 / 2}$ and $L \sim t^{2 /(2+\lambda)}$, and conclude that the random walk dominates the drift; hence, the diffusion-controlled behavior, $n \sim t^{-d / 4}$, is expected. Thus, the regime described by the lower line of Eq. (10) does not appear for $d \geq 2$. Note that for truly Coulomb systems, $\lambda=d-1$, the scaling prediction of Eq. (10) is $n \sim t^{-1} ; i$. e., the classical kinetic law. This is expected to arise when $d / 2 \leq \lambda=d-1 \leq 2$, i. e., $2 \leq d \leq 3$. For $d<2$, size-dependent kinetics is anticipated; while for $d>3$, the Coulomb interaction becomes irrelevant and the diffusion-controlled behavior emerges. Another interesting example is the system of $D$-dimensional Coulomb charges confined to the hypersurface; i. e., $\lambda=d=D-1$. In this case, Eq. (10) predicts logarithmic corrections to the power law behavior.

However, it is not clear whether the concept of unpenetrable (untransparent) domains with continuous boundaries is still applicable for $d>1$. Competition between screening, which makes long-range interaction effectively short-range, and annihilation may also significantly affect the behavior of the system. We attempted to simulate a $2 d$ system on a 
lattice. Either because of the large effective diffusion, which is inevitably introduced by the discrete nature of the lattice model, or for some deeper reason, we were unable to observe any scaling-like behavior. Since many-dimensional continuous many-body simulations are still computationally challenging, we leave this problem for the future.

More can probably be done with one-dimensional systems as well. One can try to find exact results for some specific values of the force exponent $\lambda$. For the Coulomb system, $\lambda=0$, we indeed succeeded in finding some properties analytically (see Appendix A and Ref. [11]), but we still could not find a complete solution. Another extreme case, $\lambda \rightarrow \infty$, is also theoretically challenging. (In fact, the diffusion determines the dynamics for $\lambda>4$ so one should consider a strictly noiseless system). The dynamics in the $\lambda \rightarrow \infty$ limit is extremal: One picks the pair of nearest neighbors that are closest to each other and removes it if the charges are dissimilar, or recedes them if the charges are similar. The receding is stopped when the distance reaches the second minimal intercharge distance. Then, if this second closest pair contains dissimilar charges, it is removed while the first pair continues receding; if the second pair is also the same-species, both pairs recede. If the initial sequence is alternating as it takes place with domain walls in the quench process, one always removes the closest pairs, and the domain-size distribution function approaches the scaling form. This model turns out to be completely solvable $[13-15,8]$. It would be very interesting to study a more complex version of the extremal dynamics that arises from the present two-species annihilation process.

Finally, we discuss a model where motion of the particles is ballistic; $i . e$. , it is described by Newton's laws. It is clear enough that the only change one needs to make in the above approach is to put $d^{2} L / d t^{2}$ instead of $d L / d t$ into the left hand side of (6). Assuming 
that initial velocities are irrelevant, we obtain for the concentration

$$
n(t) \sim\left\{\begin{array}{lll}
N^{\frac{2 \lambda-1}{4}} t^{-1} & \text { if } & \lambda<1 / 2, \\
t^{-\frac{2}{1+2 \lambda}} & \text { if } & 1 / 2<\lambda<1, \\
(t \log t)^{-\frac{2}{3}} & \text { if } & \lambda=1, \\
t^{-\frac{2}{2+\lambda}} & \text { if } & 1<\lambda<2, \\
t^{-\frac{1}{2}} & \text { if } & \lambda \geq 2 .
\end{array}\right.
$$

When $\lambda \geq 2$, the inertia dominates the drift and, therefore, the ballistic-controlled asymptotic behavior [16], given by the last line of Eq. (11), follows.

Numerical simulations performed for $\lambda=0.75$ showed that the concentration decays as $t^{-0.79}$ compared to $t^{-4 / 5}$ as it follows from Eq. (11).

\section{Acknowledgments}

We are thankful to S. Redner for numerous discussions. We gratefully acknowledge ARO grant \#DAAH04-93-G-0021 for partial support of this research. 


\section{APPENDIX A: $\lambda=0$}

The $\lambda=0$ case, corresponding to the "real" $1 d$ Coulomb force has several peculiar features. Since the forces between particles do not depend on the distance, and particles disappear in pairs, the net force acting on a particle is constant throughout its life. It means, that velocity of any particle is constant and equal to the difference between total charge to the left and total charge to the right of it multiplied by a charge of the particle. Taking into account that initial distribution of particles is Poissonian, one can easily describe the behavior of the system if the probability distribution for "charge imbalance" were known. To get an insight about this charge imbalance distribution, we look at our configuration of charges as on a $1 d$ random walk (RW) (Fig.3). Step up corresponds to a positive charge, step down - to the negative; since the system is overall neutral, the RW returns to the origin after $2 N$ (the size of the system) steps. The net force acting on a particle is equal to the "height" $h$, positive or negative, of the corresponding point in the RW picture. The joint distribution function $W_{2 N}(h, 2 L)$ tells us how many segments (loops in the RW terminology) of the length $2 L$, starting and ending at the "height" $h$ from the origin, exist in a RW coming to the origin (not necessarily for the first time) after $2 N$ steps. Knowing this function, one can readily calculate a life expectation time for each particle, and therefore the concentration decay rate:

$$
\frac{d n}{d t}=-\frac{1}{\tilde{L}} \sum_{L=0}^{N} \sum_{h=0}^{N-L} W_{2 N}(h, 2 L) P_{2 L}[2 t(2 h+1)]
$$

Here $\tilde{L}$ is initial system length and $P_{j}(x)=x^{j} e^{-x} / j$ ! the Poisson distribution function. So

far we have been able to determine another function, $\tilde{W}_{2 N}(2 L)$, which gives the probability that the maximum length of "zero-height" segment in a system with $2 N$ charges is $2 L$ [11]. This maximum length segment determines the lifetime of the whole system, which is 
shown to be proportional to its size $2 N$. Moreover, the life time distribution function has a remarkably rich structure (an infinite set of singularities, etc.; see [11]).

Although we were not able to find the exact expression for the decay of concentration in this case, numerical simulations of this problem prove to be very simple. Instead of running a molecular dynamics algorithm, it is sufficient to calculate all the net forces once and find an annihilation partner for each particle; after that, we know the lifetimes for all the particles in the system. It enabled us to check an accuracy of our molecular dynamics simulation for our standard $\left(10^{4}\right.$ pairs $)$ system size and also to study the systems with up to $10^{5}$ particles of each species. Even for these relatively big systems, we were unable to find any power-law behavior; the function that fits best our simulation results looks like $n(t) \sim \exp \left(-0.05 \ln ^{4} t\right)$

\section{APPENDIX B:}

\section{TYPICAL FORCE IN A SYSTEM OF CHARGED PARTICLES}

A problem of distribution of a typical force in a system of particles with Coulomb interaction was first studied by Holtsmark [10]. Using the same approach, we will show, that for arbitrary dimensionality and $r^{-\lambda}$ interaction, the typical (or mean) force is finite in an infinite system only for some range of the force constants $\lambda$, specifically for $\lambda>d / 2$. We start from an expression for force distribution function $W(F)$, which gives the probability that the force, acting on a "test particle" which we will put at the origin, is equal to $F$ :

$$
W(\vec{F})=\left\langle\delta\left(\vec{F}-\sum_{j=1}^{N} \vec{f}\left(\vec{r}_{j}\right)\right)\right\rangle_{a v}
$$

where $\vec{f}\left(\vec{r}_{j}\right)=\vec{r}_{j} / r_{j}^{\lambda+1}$ is the force exerted by a $j^{\text {th }}$ particle on the test one. Since we assume that spatial distribution of particles is random and independent, it is sufficient to 
consider one-component system. Replacing $\delta$-function by auxiliary integration over $d \vec{k}$ we obtain:

$$
W(\vec{F})=\frac{1}{(2 \pi)^{d}} \int \exp (i \vec{k} \cdot \vec{F}) S(\vec{k}) d \vec{k}
$$

with

$$
S(\vec{k})=\int \ldots \int \exp \left(-\sum_{j=1}^{N} i \vec{k} \cdot \vec{f}\left(\vec{r}_{j}\right)\right) \prod_{j=1}^{N} \frac{d \vec{r}_{j}}{V} \equiv\left(\frac{1}{V} \int e^{-i \vec{k} \cdot \vec{f}} d \vec{r}\right)^{N} .
$$

Here $\vec{f}=\vec{r} / r^{\lambda+1}, N$ is the number of particles in the system, and $V$ is the volume. Rewriting (B3) in the form

$$
S(\vec{k})=\left[1-\frac{1}{V} \int\left(1-e^{-i \vec{k} \cdot \vec{f}}\right) d \vec{r}\right]^{N}
$$

and taking the thermodynamic limit, $N \rightarrow \infty$ and $V \rightarrow \infty$ where $N / V=n$ is kept fixed, yields

$$
S(\vec{k})=\exp \left(-n \int\left[1-e^{-i \vec{k} \cdot \vec{r} / r^{\lambda+1}}\right] d \vec{r}\right)
$$

After expanding the exponent in the integrand for large $r$ and performing angular integration (which eliminates all odd-order terms) one finds that for system size $R \rightarrow$ $\infty$, the integral in the right-hand side of Eq. (B5) converges only if $\lambda>d / 2$ while for $\lambda<d / 2$ the integral diverges with the system size. More precisely, a considerable but straightforward computation yields

$$
S(\vec{k}) \equiv S(k)=\exp \left[-n \Omega_{d} A(d, \lambda) k^{\frac{d}{\lambda}}\right]
$$

for $\lambda>d / 2$. In Eq. (B6), $\Omega_{d}=2 \pi^{d / 2} / \Gamma(d / 2)$ denotes the surface area of the unit sphere in $d$ dimensions and $A(d, \lambda)$ is the shorthand notation for the integral

$$
A(d, \lambda)=\int_{0}^{\infty} \frac{d z}{\lambda z^{\frac{d}{\lambda}+1}}\left[1-\Gamma\left(\frac{d}{2}\right)\left(\frac{2}{z}\right)^{\frac{d}{2}-1} J_{\frac{d}{2}-1}(z)\right]
$$


with $\Gamma(z)$ being the Euler gamma function, and $J_{\nu}(z)$ the Bessel function. For $\lambda<d / 2$ the integral in the left-hand side of Eq. (B5) grows as $\frac{2 \Omega_{d}}{d(d-2 \lambda)} R^{d-2 \lambda} k^{2}$. Returning back to Eq. (B2) we see that in the case of $(\lambda \leq d / 2)$ the net force is given by

$$
W(\vec{F})=\frac{1}{(2 \pi)^{d}} \int \exp \left(i \vec{k} \cdot \vec{F}-\frac{2 \Omega_{d}}{d(d-2 \lambda)} R^{d-2 \lambda} k^{2}\right) d \vec{k}
$$

In the limit $R \rightarrow \infty$, we compute the integral in Eq. (B7) asymptotically to find

$$
W(\vec{F}) \sim\left[(d-2 \lambda) R^{2 \lambda-d} F\right]^{d-1} \exp \left[- \text { const }(d-2 \lambda) R^{2 \lambda-d} F^{2}\right] .
$$

Eqs. (B7) and (B8) are valid for $\lambda<d / 2$. For $\lambda=d / 2, R^{d-2 \lambda} /(d-2 \lambda)$ should be replaced by $\log R$. For $d=1$ and $R \propto N$, the net force has the Gaussian distribution, $W(\vec{F}) \propto(1-2 \lambda)^{1 / 2} N^{\lambda-1 / 2} \exp \left[-(1-2 \lambda) N^{2 \lambda-1} F^{2}\right]$, and therefore the typical force grows with size as $N^{1 / 2-\lambda}$ in agreement with qualitative results of Sec. III. Note also that for $\lambda=1 / 2$ the typical force still grow with system size, $F \sim \sqrt{\log N}$, and hence the density decay of the form $n(t) \sim(\log N)^{-1 / 4} t^{-1 / 2}$ is expected. 


\section{References}

1. For a recent review, see S. Redner and F. Leyvraz, in Fractals in Science, eds. A. Bunde and S. Havlin (Springer-Verlag, Berlin, 1994).

2. T. Ohtsuki, Phys. Lett. A 106, 224 (84).

3. V. Kuzovkov and E. Kotomin, J. Stat. Phys. 72, 127 (1993).

4. M. Mandello and N. Goldenfeld, Phys. Rev. A 42, 5865 (1990).

5. W. G. Jang, V. V. Ginzburg, C. D. Muzny, and N. A. Clark, Phys. Rev. E 51, 411 (1995).

6. F. J. Dyson, Commun. Math. Phys. 12, 91 (1969).

7. B. P. Lee and J. L. Cardy, Phys. Rev. E 48, 2452 (1993).

8. A. D. Rutenberg and A. J. Bray, Phys. Rev. E 50, 1900 (1994).

9. T. Ohta and H. Hayakawa, Physica A 204, 482 (1994).

10. See, e. g., S. Chandrasekhar, Rev. Mod. Phys. 15, 1 (1943).

11. L. Frachebourg, I. Ispolatov and P. L. Krapivsky, unpublished.

12. L. Greengard, The Rapid Evaluation of Potential Fields in Particle Systems, (MIT Press, Cambridge, 1988).

13. T. Nagai and K. Kawasaki, Physica A 134, 483 (1986); K. Kawasaki, A. Ogawa, and T. Nagai, Physica B 149, 97 (1988).

14. A. J. Bray, B. Derrida, and C. Godreche, Europhys. Lett. 27, 175 (1994); A. J. Bray and B. Derrida, Phys. Rev. E 51, 1633 (1995).

15. S. N. Majumdar and D. A. Huse, Phys. Rev. E 52, 270 (1995).

16. Y. Elskens and H. L. Frisch, Phys. Rev. A 31, 3812 (1985). 


\section{Figure Captions}

Figure 1. Schematic illustration of domain structure. Typical domain has length $L$ and consists of $M=6$ particles, average distance between particles is $R=L / N$.

Figure 2. Plot of concentration $n(t)$ vs. time on a double logarithmic scale for $\lambda=1(\diamond), \lambda=$ $3 / 4(\triangle), \lambda=1 / 2(\nabla) \lambda=1 / 4(\bigcirc)$, and $\lambda=0(\star)$.

Figure 3. RW representation of a two species neutral system. Number of particles of each species $N=17$. A neutral segment of the length $2 L=16$ is shown having $h=2$ uncompensated charges to the left and to the right of it. 